\title{
Face Recognition using Fast GA
}

\author{
B.S.Sathish, A Daveedu Raju, G. Prasanth Kumar, P.V.K Kumar, Y. Nagendra Kumar
}

\begin{abstract}
Face Identification System using a fast genetic algorithm computation (FGA) is presented. FGA is used to compute and search the face in a database. The objective of the work is to make a face identification system which can recognize face from a given image or any other image streaming system like webcam. The system also has to detect the face from a system accurately in order to identify the face accurately. The image can be captured either from a proposed webcam or a captured JPEG or PNG image or any other data source. The system needs training with adequate sample images to perform this operation. Training the generic system plays a vital role in identifying the face in an image. A tolerance is identified as a limit to the genetic algorithm which acts as a terminal condition to the evolution. A unique encoding is used which stores the facial features of a human face into numeric string which can be stored and searched with much ease thereby decreasing the search and computational time. Template matching technique is applied to identify the face in a big picture. Generation of an Eigen face is obtained by the stage a mathematical practice called PCA. Eigen Features is also computed such that the measurement of facial metrics is done using nodal point measurement.
\end{abstract}

Keywords: Computational Time, Eigen face, Fast Genetic Algorithm, Face Identification, Nodal Point Measurement, Principal Component Analysis.

\section{INTRODUCTION}

The difficulty in real-time detection of face and its features of such an application is that the identification is not as accurate enough and acutely time consuming. The detection of a single face in today's available technology and applications are nearly close enough to be accurate to a level of $65 \%$. This work utilizes the evolutionary computational methods and image processing capability to sense and detect the human face. Proposed work have chosen Genetic Algorithm to be our choice of technology because the biological nature of the problem statement. Human face is biometrically unique and has a special feature developed by chromosomes and DNAs. So Proposed work use the same technology to find the nearest of the available results.

Proposed work use a specialized technology called fast genetic algorithm to shorten the length of the computation. The main disadvantage of the genetic or evolutionary

Revised Manuscript Received on November 22, 2019

Dr.B.S.Sathish, Dept of ECE, Ramachandra College of Engineering, Eluru, India. Email: subramanyamsathish@yahoo.co.in

Dr. A Daveedu Raju, Dept of CSE, Ramachandra College of Engineering, Eluru, India. Email: davidrecharge@ gmail.com

G. Prasanth Kumar, Dept of CSE, Ramachandra College of Engineering, Eluru, India. Email: prasanthg.cse@ gmail.com

P.V.K Kumar, Y. Nagendra Kumar, Dept of CSE, Ramachandra College of Engineering, Eluru, India. Email: kishore.rce@ gmail.com, nagendrayakkala@gmail.com computation is that the terminal condition is not clearly defined in the application. Proposed work use the fast genetic algorithm which settles to a definite threshold point of the derivative result set. This drastically reduces the length and narrow down of the computation to a specific result as shown in figure 1.
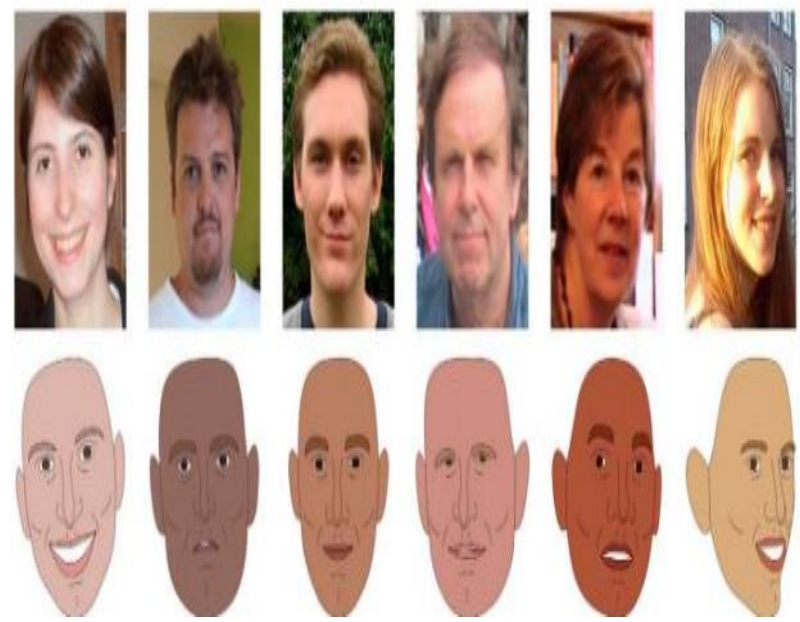

Fig. 1. Face Reconstruction using Modeled Attributes

\section{ORIGIN OF THE PROBLEM}

Face detection is the initial, simple and the most important step of all the other steps in the overall system. We involve 2 entirely different tools on the same platform in order to accomplish the necessary step in the whole process of face identification. The Important tools used in the image acquisition and processing are Java Media Framework (JMF) and OpenCV (Open Source Computer Vision Library). Acquisition of image and other media operations like filtering and sub selection of image is done by Java Media Framework (JMF). This work also utilizes OpenCV System to detect the face from the captured image as shown in figure 2. From the image shown in above figure, it is observed that various steps in the face acquisition and detection do various tasks. In the initial step, we see that the JMF captures the image from the camera or the provided webcam. The captured image is then detected and cropped by digital algorithms and image processing algorithms which are customized from computer vision library. 


\section{Face Recognition using Fast GA}

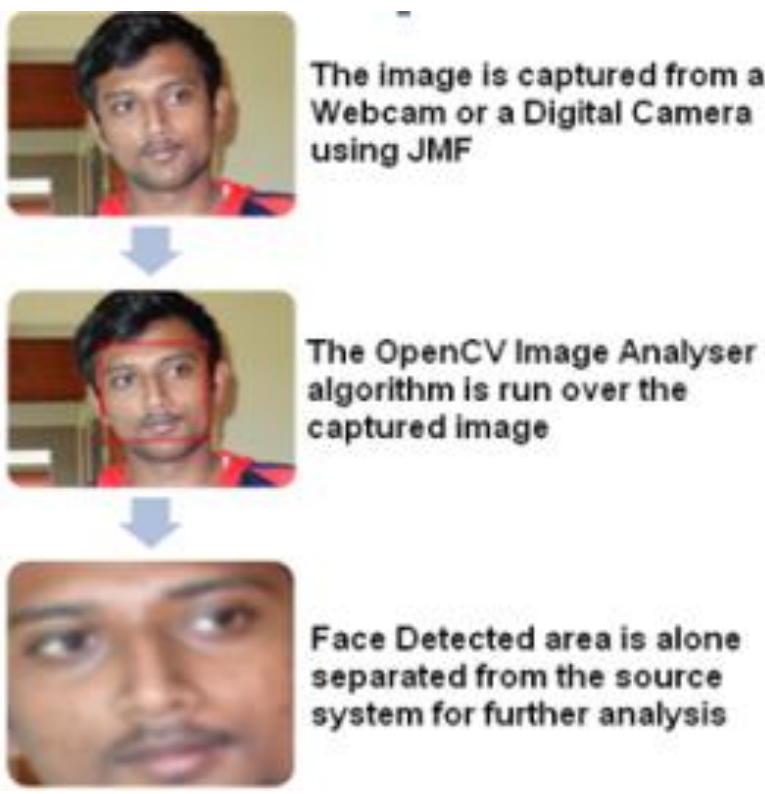

Fig. 2. Steps of Face Detection Overview

\section{A. Face Alignment}

Face Alignment is the second step in identification of the Human face from a compound digital image. In this step we follow two important steps which measure the distinct parameters of a face. The tools used for this process are template matching and Image filtering. These two are much important because these tools help to remove any unnecessary details in an image and also help to align the image towards the best fit result from the system as shown in figure $3 a, 3 b$.

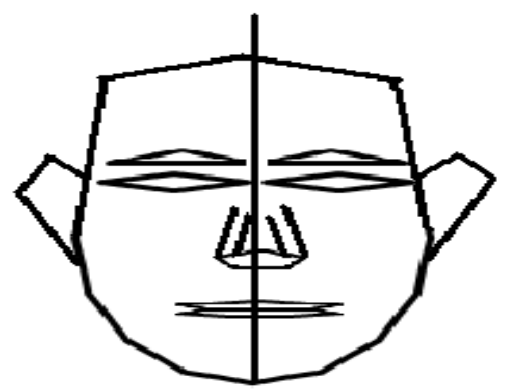

Fig. 3(a). Vector full feature template

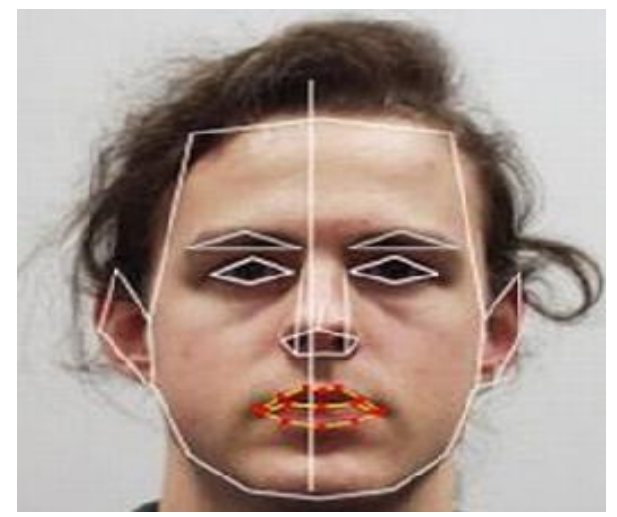

Fig. 3(b). Sample Image

\section{B. Image Filters}

There are certain unnecessary features available in the image for the application of face detection, such as color, texture, contrast, brightness, image grains, and so on. These unnecessary details must be filtered such that these don't create any unnecessary deviation from the desired result. So the image is passed through a series of handmade filters which are general purpose filters customized to suit this specific need. The captured image is run through the following filters.

1. Skin Color Filter

2. Edge Filter

3. Grayscale Filter

4. Invert Filter.

Refer figure 4 for results obtained at the end of each filters. With the help of these filters, we obtain a metadata Specifics which is more of detailed information on the features rather than emphasizing on the unnecessary details such as color, contrast and other unnecessary deviation. Fig 4 shows various filters being used on sample images. The actions of the filters are described step by step as used by the software to detect face. The major intend of the sort is sieve and emboss edges of available scheme. This proves an advantage to reduce the unnecessary details in an image such as the background, details, lighting mode, angle of the lighting, shadow etc., as shown in figure 4.

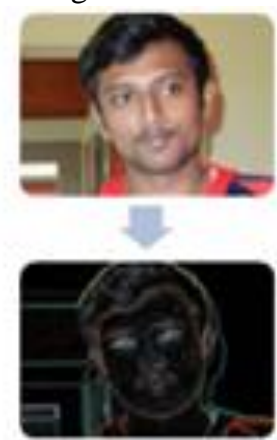

\section{Raw Captured Image}

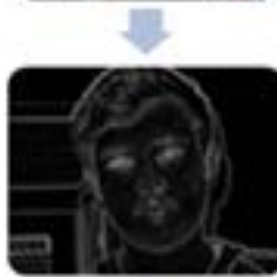

Edge Detection Filter

Gray Filter

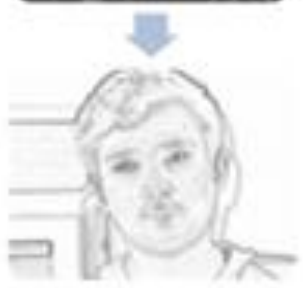

Invert Filter

Fig. 4. Steps of Image Filter Results

\section{Nodal Point Measurement}

The capability of individual to recognize other people's faces, and often put a name and complete identity history to them, is truly amazing. In reality it is so good quality that be apt to take it very much for granted and don't really appreciate how sensitive we are to small variations in appearance. Humans have evolved an ability to recognize other humans in difficult situations a glimpse of a face in the crowd is often enough for a positive recognition as shown in figure 5 .

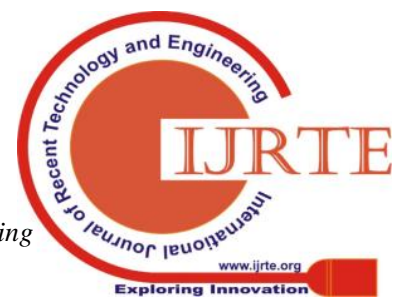




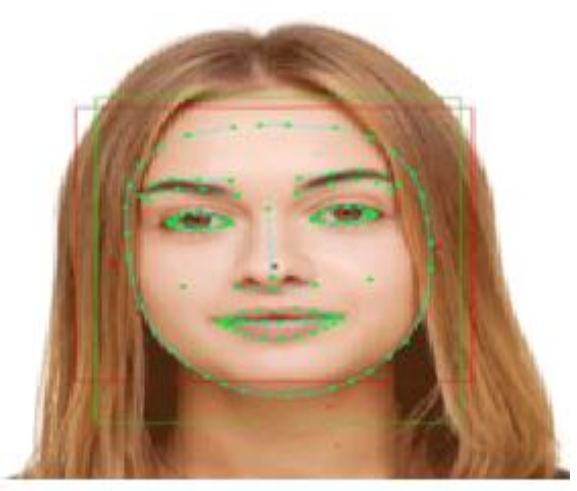

Fig. 5. Nodal Point Measurement

\section{RESULT AND DISCUSSION}

The use of this fast genetic algorithm is based on a research paper. The results of this were promising that the system was holding good with the quicker matching by the use of tolerance level being defined. There was also an API Library available for the general usage which is used to obtain quicker results by referring to the previous researches. The Fast Genetic Algorithm is a System which obtains a terminal point for the evolutionary model of the results obtained by creating a terminal condition. This Terminal condition is having a threshold of closeness to result. A threshold percentage is created which will clearly define the terminal point for the evolutionary process. The Human brain acts in such a way that it receives a partial data from the source while it does a parallel processing to search the image from the memory. We use the same technique to fetch in a faster mode. We use this clustered search in a fast genetic style in-order to satisfy the search time of the System. In-order to do that, we introduce a terminal point to the system which acts as a level restrictor for a genetic search algorithm which stops the tree based implementation of the evolutionary nature at certain point such that the system arrives at a specified result set of images which is meant to be filtered out manually. Here we use the precast library prepared by the Fast GA to retrieve the details available in the Genetic Algorithm based Search engine. This Search is optimized at a prior level and is utilized to adopt to be used in the system as shown in figures $6,7,8,9$.

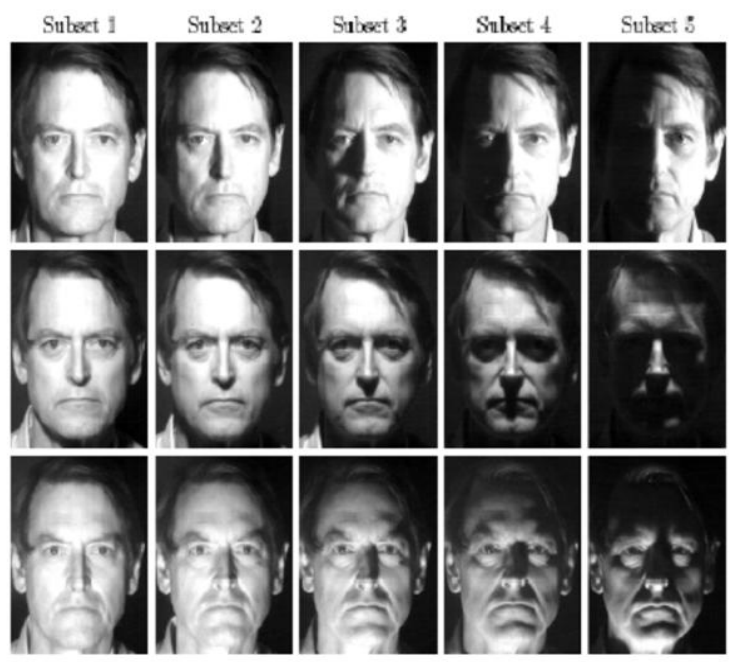

Fig. 6. Fast Genetic Algorithm Cluster

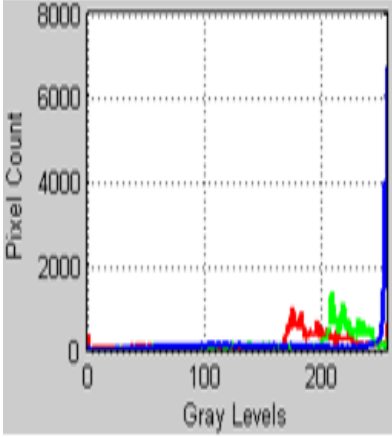

(a)

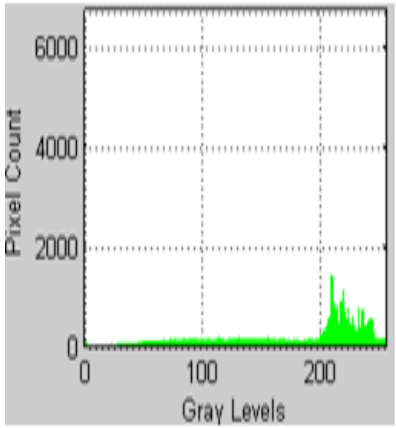

(c)

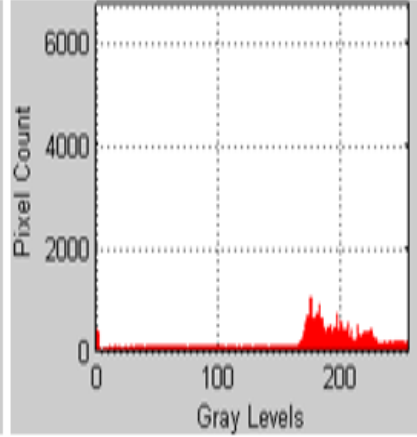

(b)

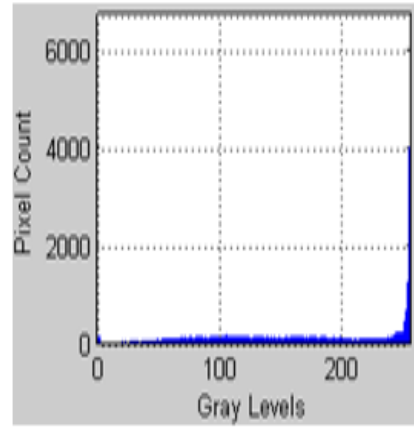

(d)

Fig. 7. Histogram Representation of Fast Genetic Algorithm Cluster

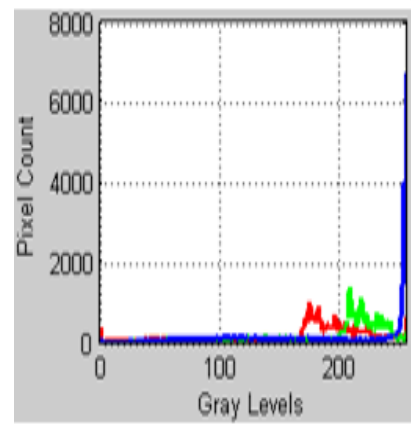

(a)

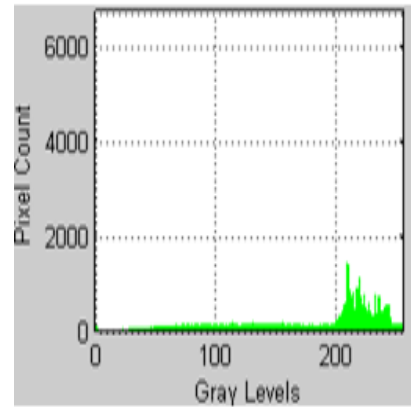

(c)

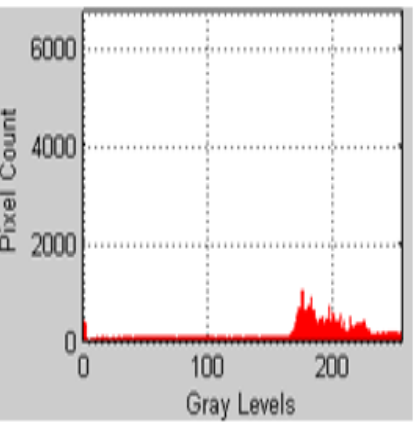

(b)

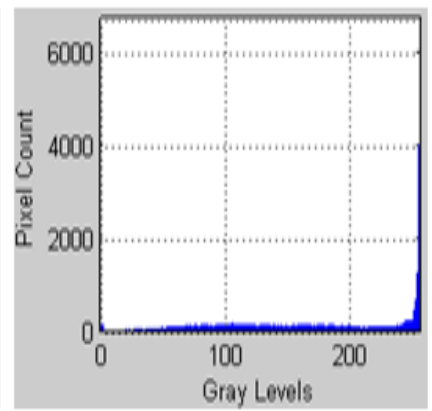

(d)

Fig. 8. Fast Genetic Algorithm Cluster Eigen Faces

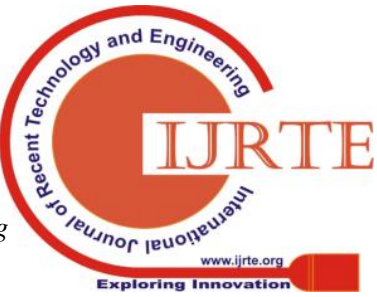




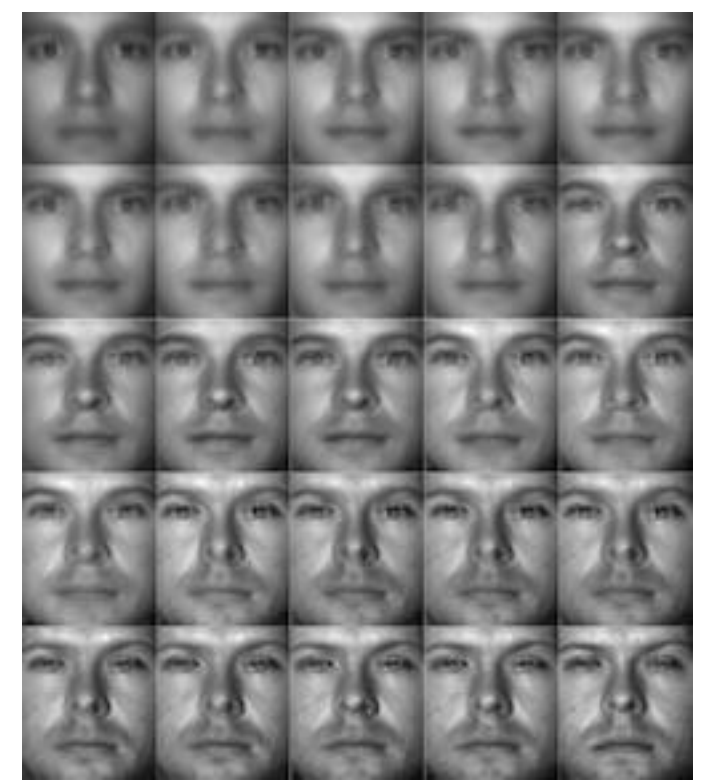

Fig. 9. Histogram Representation of Fast Genetic Algorithm Cluster Eigen faces

\section{CONCLUSION}

There is a very huge scope of face recognition in the market today. We do not have a biometric face search algorithm with highest accuracy which is a major challenge in today's engineering world. Face recognition has long been a goal of computer vision, but only in recent years reliable automated face recognition has become a realistic target of biometrics research.

\section{REFERENCES}

1. P. Ganesan and V. Rajini, "A method to segment color images based on modified Fuzzy-Possibilistic-C-Means clustering algorithm," Recent Advances in Space Technology Services and Climate Change 2010 (RSTS \& CC-2010), IEEE, Chennai, 2010, pp.157-163, 2010.

2. worknshuo Gao; Xiaoguang Zhang; Lei Yang; Huizhong Liu, "An improved Sobel edge detection," Computer Science and Information Technology (ICCSIT), 2010 3rd IEEE, vol.5, no 1, 2010.

3. Kiani, A.; Sahebi, M.R., "Edge detection based on the Shannon Entropy by piecewise thresholding on remote sensing images," in Computer Vision, IET, vol.9, no.5,pp.758-768, 2015.

4. Ganesan, P., and V. Rajini. " Segmentation and denoising of noisy satellite images based on modified fuzzy c means clustering and discrete wavelet transform for information retrieval", International journal of engineering and technology, vol.5, no.5, 2013, pp.3856-3869, 2013.

5. Melin, P.; Gonzalez, C.I.; Castro, J.R.; Mendoza, O.; Castillo, O., "Edge-Detection Method for Image Processing Based on Generalized Type-2 Fuzzy Logic," in Fuzzy Systems, IEEE Transactions on , vol.22, no.6, pp.1515-1525, Dec.2014.

6. Caixia Deng; Proposed workifeng Ma; Yin Yin, "An edge detection approach of image fusion based on improved Sobel operator," Image and Signal Processing (CISP), 2011, vol.3, no., pp.1189,1193, 15-17 Oct. 2011.

7. Shaik, K.B.,Ganesan, P.,Kalist, B.S.,Sathish, B.S.,Jenitha, J.M.M,' Comparative Study of Skin Color Detection and Segmentation in HSV and YCbCr Color Space “, Procedia Computer Science, 57 ( 2015 ), pp. $41-48,2015$.

\section{AUTHORS FROFILE}

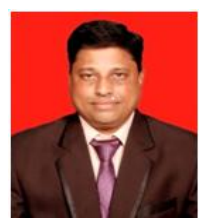

Dr.B.S.Sathish, Professor / Research Head, Department Of Electronics And Communication Engineering, Ramachandra College Of Engineering, Eluru, India. 